\title{
Full two-photon down-conversion of a single photon
}

\author{
E. Sánchez-Burillo, ${ }^{1}$ L. Martín-Moreno, ${ }^{1}$ J. J. García-Ripoll, ${ }^{2}$ and D. Zueco ${ }^{1,3}$ \\ ${ }^{1}$ Instituto de Ciencia de Materiales de Aragon and Departamento de Fisica de la Materia Condensada, \\ CSIC-Universidad de Zaragoza, E-50012 Zaragoza, Spain \\ ${ }^{2}$ Instituto de Fisica Fundamental, IFF-CSIC, Calle Serrano 113b, Madrid E-28006, Spain \\ ${ }^{3}$ Fundacion ARAID, Paseo Maria Agustin 36, E-50004 Zaragoza, Spain
}

(Received 7 April 2016; published 9 November 2016)

\begin{abstract}
We demonstrate, both numerically and analytically, that it is possible to deterministically generate two photons from one and only one photon. We characterize the output two-photon field and make our calculations close to reality by including losses. Our proposal relies on real or artificial three-level atoms with a cyclic transition strongly coupled to a one-dimensional waveguide. We show that almost perfect down-conversion, with efficiency over $99 \%$, is reachable using state-of-the-art waveguide QED architectures such as photonic crystals or superconducting circuits. In particular, we sketch an implementation in circuit QED, where the three-level atom is a transmon.
\end{abstract}

DOI: 10.1103/PhysRevA.94.053814

\section{INTRODUCTION}

The interaction between the electromagnetic field and quantum discrete level systems (like atoms) may be enhanced by confining light in one-dimensional waveguides [1-12]. In these setups, a key parameter is the ratio between the decay rate due to coupling to waveguide photons and that due to coupling to all other channels. Whenever the former dominates, we are in the strong-coupling regime of light-matter interactions. In this case, a single two-level system can be used not only to induce effective photon-photon interactions, but it also enables minimal and highly efficient optical devices, such as perfect mirrors [13-15], single-photon lasing [16], and Raman scattering [17-19].

Another optical process that could strongly benefit from an enhanced light-matter interaction is photon down-conversion, where a light beam of a given frequency is split into two beams whose frequencies add up to the original one. Downconversion is routinely used for the generation of entangled photons and of light at convenient frequencies. This is already done in atomic and molecular systems and it could also be useful for energy harvesting, by using photons of high energy to excite more suitable transitions in a photovoltaic material. Photon down- and up-conversion are currently realized in bulk optics with the help of nonlinear noncentrosymmetric materials [20]. However, due to the smallness of the fine structure constant, the typical performance of this process in crystals such as beta barium borate crystals is very small, with only about one in every $1 \times 10^{12}$ photons being downconverted [20].

A cyclic three-level system (C3LS) strongly coupled to a waveguide is the minimal setup that produces downconversion. When classical light is used as input, only a small part of the incident power is converted into a correlated twophoton output field [21-23]. In chiral waveguides, however, it has been argued that two photons can be generated when one and only one photon is scattered in a C3LS structure [24]. Another down-conversion mechanism at the single-photon limit, requiring the driving of nonlinear cavities, was recently proposed [25]. In this paper we generalize the results in the C3LS, considering full down-conversion efficiency in nonchiral waveguides. More precisely, we consider a waveguide photon impinging on the C3LS and resonantly populating level $|2\rangle$, as schematically represented in Fig. 1(a). In addition to the direct relaxation of $|2\rangle$ to the ground state, the cascade $|2\rangle \rightarrow|1\rangle \rightarrow|0\rangle$ allows the relaxation to be accompanied by the emission of two photons [26]. In our study we include losses, analyze the entanglement of the output field, and suggest a possible experimental realization.

The rest of the paper is organized as follows. In the next section, we introduce the model. In Sec. III we sketch a realization in circuit QED. We continue in Sec. IV by reporting our numerical results, based on matrix product states (MPSs). There, we discuss the down-conversion probability and the dynamics for both the field and the atom. We also characterize the output field and its entanglement. In Sec. V we develop an analytical theory, which gives the efficiency for down-conversion in the presence of losses (Sec. VI). Section VII is devoted to the conclusions and we develop some technical issues in four Appendices.

\section{MODEL}

We consider a cyclic three-level quantum system (C3LS) strongly coupled to a one-dimensional waveguide where photons can travel freely. We neglect thermal fluctuations and losses in the waveguide and, for the moment, in the C3LS, so the effective Hamiltonian is $(\hbar=1)$

$$
H=H_{0}+H_{\text {int }},
$$

where

$$
H_{0}=\int d \omega \omega r_{\omega}^{\dagger} r_{\omega}+\int d \omega \omega l_{\omega}^{\dagger} l_{\omega}+\sum_{j=0}^{2} \Omega_{j}|j\rangle\langle j|,
$$

with $r_{\omega}$ and $l_{\omega}$ being bosonic operators that, respectively, annihilate right- and left-moving waveguide photons; $r_{\omega}^{\dagger}$ and $l_{\omega}^{\dagger}$ are the corresponding creation operators. Besides, $\Omega_{j}$ and $|j\rangle$ are the eigenenergies and eigenstates of the isolated three-level system (3LS). The coupling between the 3LS and the waveguide photons is represented by $H_{\text {int }}=G X$, with $X$ the electromagnetic (EM) displacement given by

$$
X=\int d \omega D(\omega)\left(r_{\omega}+l_{\omega}\right)+\text { H.c. }
$$


(a)

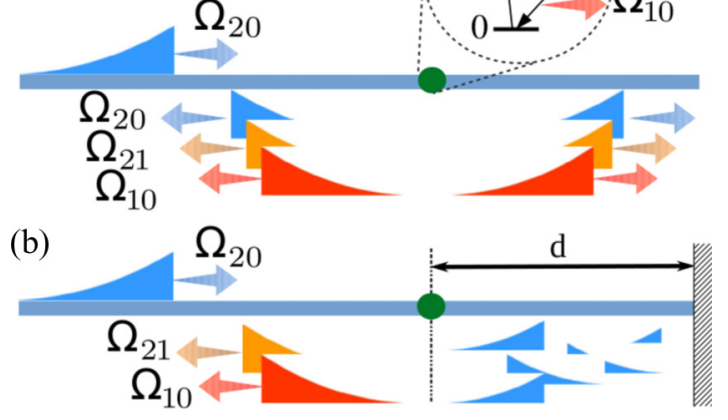

FIG. 1. Down-conversion setup. (a) A single incoming photon interacts with a three-level system. Part of it is elastically transmitted or reflected $\left(\Omega_{20}\right.$, blue) and part is down-converted into a pair of photons with frequencies $\Omega_{21}$ and $\Omega_{10}$ (orange and red). (b) Placing a mirror right after the scatterer at a suitable distance $d$, down-conversion can become deterministic: all reflected photons have down-converted frequencies.

where $D(\omega)$ is the density of states. The operator $G$ accounts for the transitions between levels in the C3LS induced by the EM field. Its matrix elements are $\langle i|G| j\rangle \equiv G_{i j}$.

\section{A POSSIBLE IMPLEMENTATION}

An important point is that a C3LS cannot be realized in systems (i) that are so small compared to the waveguide that the dipolar interaction dominates (like atoms) and (ii) whose quantum states are labeled by a spatial parity tag. The reason is that at least two of the three states in the C3LS must have the same parity, but the dipole interaction only couples states with different parity. However, effective C3LSs may appear in extended quantum systems, where couplings beyond the dipolar term must be considered. Implementations of the C3LS are some molecules [27] and flux qubits made of superconducting circuits [21,22]. Nevertheless, this last system leads to three quite dissimilar excitation energies. In order to obtain two down-converted photons of similar (and possibly equal) frequencies, we consider an alternative design for an effective C3LS in the microwave range using a transmon (a charge superconducting qubit shunted by a big capacitor) [28].

Typically, inductive coupling between the transmon and the transmission line is negligible. The reason is that the transmon design is basically that of a one-dimensional electric dipole, without support for currents. In addition to this, the superconducting quantum interference device (SQUID) that controls the transmon frequency is small and shielded away from any coupling with the transmission line. Inductive couplings between transmons have been demonstrated, however [29,30]. We make use of similar ideas to envision a different coupling architecture that allows one to break the parity symmetry in the transmon setup.

Our starting point is a setup such as the one in Fig. 2(a), where the transmon SQUID is no longer screened and the superconducting island couples both capacitively and

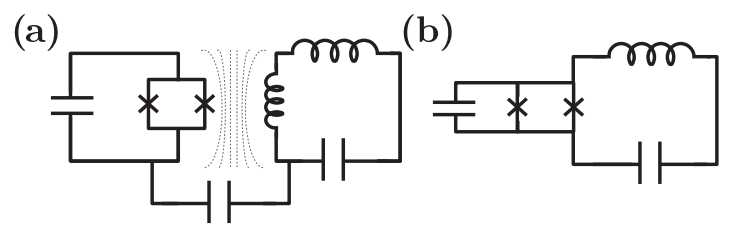

FIG. 2. (a) A transmon can be both inductively and capacitively coupled to an LC resonator. Coupling strength can be increased by either increasing the SQUID area or (b) sharing a conductor segment, in the spirit of Ref. [31] and similar proposals.

inductively to the resonator. The circuit Lagrangian (with inductive and capacitive coupling) is

$$
\begin{aligned}
\mathcal{L}= & \int d x c\left(\partial_{t} \phi(x, t)\right)^{2}-\frac{1}{l}\left(\partial_{x} \phi(x, t)\right)^{2} \\
& +\frac{1}{2 C_{\Sigma}}(q-\mathcal{Q})^{2}-E_{J} \cos \left(2 \pi \Phi / \Phi_{0}\right) \cos \varphi .
\end{aligned}
$$

The first line accounts for the transmission line Lagrangian while the transmon and its coupling are written in the second line. Here, $\phi(x, t)$ is the (quantum) flux field, which in the interaction picture reads

$$
\begin{aligned}
\phi(x, t)= & \sqrt{\frac{\hbar Z_{0}}{4 \pi}} \int_{0}^{\infty} d \omega \frac{1}{\sqrt{\omega}}\left(r_{\omega} e^{-i \omega(t-x / v)}\right. \\
& \left.+l_{\omega} e^{-i \omega(t+x / v)}+\text { H.c. }\right),
\end{aligned}
$$

with $c(l)$ being the capacitance (inductance) per unit length and $Z_{0}=\sqrt{l / c}$ being the line impedance. The superconducting Josephson energy is $E_{J}$ and $C_{\Sigma}$ is the capacitance. Charge and phase invariant gauge are quantized via $\left[e^{i \varphi}, q\right]=2 e e^{i \varphi}$. The transmon is driven and coupled to the line via the charge $\mathcal{Q}$ and the flux $\Phi\left(\Phi_{0}=h / 2 e\right.$ is the flux quantum):

$$
\begin{gathered}
\mathcal{Q}=2 e n_{g}+c \partial_{t} \phi(x, t) \\
\Phi=\lambda \partial_{x} \phi(x, t)+\frac{\Phi_{0}}{2 \pi} \varphi_{\mathrm{ext}} .
\end{gathered}
$$

We have introduced the coupling factor $\lambda$ that accounts for the effective field by the transmon's SQUID after taking into account the screening. Inserting the latter in Eq. (4) and expanding the cosine up to first order in the quantum fluctuations $\partial_{x} \phi$ around $\partial_{x} \phi=0$, we get the coupling Hamiltonian,

$$
H_{\text {coupling }}=\frac{c}{C_{\Sigma}} q \partial_{t} \phi-\lambda d \frac{\pi}{\Phi_{0}} E_{J} \sin \left(\varphi_{\text {ext }}\right) \cos (\varphi) \partial_{x} \phi .
$$

We still need to show that Eq. (8) provides the cycliccoupling structure. We numerically diagonalize $H_{\text {transmon }}=$ $\frac{1}{2 C_{\Sigma}} q^{2}-E_{J} \cos \varphi$ in the charge basis, retaining the first three levels $H_{\text {transmon }}=\sum_{j=0}^{2} \Omega_{j}|j\rangle\langle j|$. In the basis of these three eigenstates, we can compute the different contributions to $G$ in $H$ [Eq. (1) in the main text]. In Fig. 3 we plot the contributions due to the charge operator $q$ in Eq. (8). As already explained in the literature, $\langle i|q| i+1\rangle \neq 0$ but $\langle 0|q| 2\rangle=0$ [28]. A nonzero $G_{02}$ value is obtained through the inductive coupling. Figure 3 also renders the dependence of $\langle 0|\cos (\varphi)| 2\rangle \neq 0$ on $E_{C} / E_{J}$ (notice that $\langle i|\cos (\varphi)| i+1\rangle=0$ since $\cos (\varphi)$ is an even operator and $|i\rangle$ and $|i+1\rangle$ have opposed parity). 


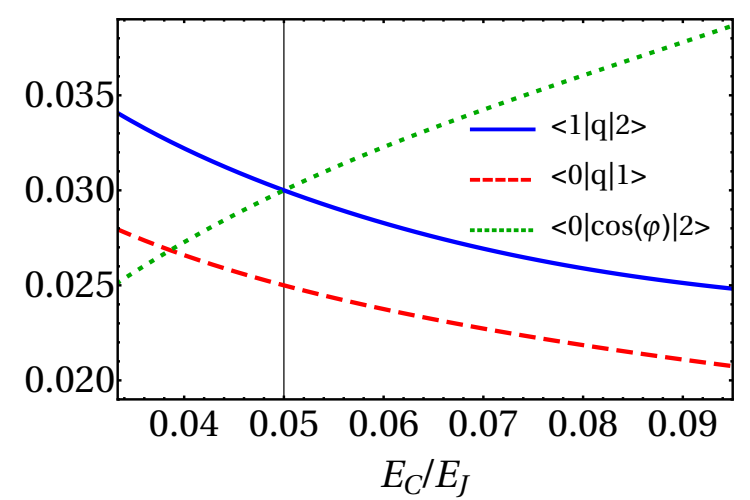

FIG. 3. Nonzero charge $\langle i|q| j\rangle$ and flux $\langle i|\cos \varphi| j\rangle$ matrix elements contributing to the coupling operator $G$ [see Eq. (8)]. The vertical line marks the parameters chosen in our simulations with $E_{C} / E_{J}=1 / 20$.

Therefore, by combining inductive and capacitive (electric and magnetic) coupling, the transmon has a cyclic structure. Through the main text we set $E_{C} / E_{J}=1 / 20$, which is a typical experimental value for transmons. This ratio, together with the density of states of the line, fixes the ratio $\Gamma_{10}^{(0)} / \Gamma_{21}^{(0)}$, with $\Gamma_{i j}^{(0)} \equiv 2 \pi D^{2}\left(\Omega_{i j}\right) G_{i j}^{2}$ being the rates between quantum levels induced by coupling to the waveguide photons. Standard experimental values are of the order of $1 \times 10^{-3} \Omega_{10}$, with $\Omega_{10} \equiv \Omega_{1}-\Omega_{0}$. Finally, we fix $\lambda$ and $C_{\Sigma}$ for making optimal for the two-photon generation (see below).

\section{NUMERICAL SOLUTION}

We numerically compute the time evolution of an initial single-photon wave packet. Our simulations assume that the incident photon was generated via spontaneous emission in an auxiliary qubit. In two-level systems the emitted wave packets are exponentially decaying functions in real space (see Appendix D). Besides, we discretize both space and time and use the MPS technique, which is a well-known method for obtaining the ground state and low energy states in interacting one-dimensional systems [31-35]. The MPS technique has been applied to photon scattering in waveguides [19,36,37]. This method is especially suited for Hamiltonians like Eq. (1) that either have a nonlinear dispersion relation or, as in the considered case, do not conserve the number of excitations. It is worth emphasizing that the MPS calculations provide both field and C3LS observables at any time.

The technical details of our simulations, as the effective model used, its spectral density, and the dispersion relation for the waveguide can be found in Appendix D. We emphasize that the physical mechanisms and the consequences of this paper are given in terms of the different spontaneous emitted rates, both radiative and nonradiative, of the scatterer.

\section{A. Two-photon generation: Scattering and dynamics}

In Fig. 4 we plot the spectrum for the onephoton transmittance and reflectance, $\quad\left|t^{(1)}(\omega)\right|^{2}=$ $\lim _{t \rightarrow \infty}\left|\left\langle\Omega\left|r_{\omega} e^{-i H t}\right| \psi_{\text {in }}\right\rangle /\left\langle\Omega\left|r_{\omega}\right| \psi_{\text {in }}\right\rangle\right|^{2} \quad$ and $\quad\left|r^{(1)}(\omega)\right|^{2}=$ $\lim _{t \rightarrow \infty}\left|\left\langle\Omega\left|l_{\omega} e^{-i H t}\right| \psi_{\text {in }}\right\rangle /\left\langle\Omega\left|r_{\omega}\right| \psi_{\text {in }}\right\rangle\right|^{2}$, respectively, and the probability of emitting two photons, $P^{(2)}(\omega)$. The latter is
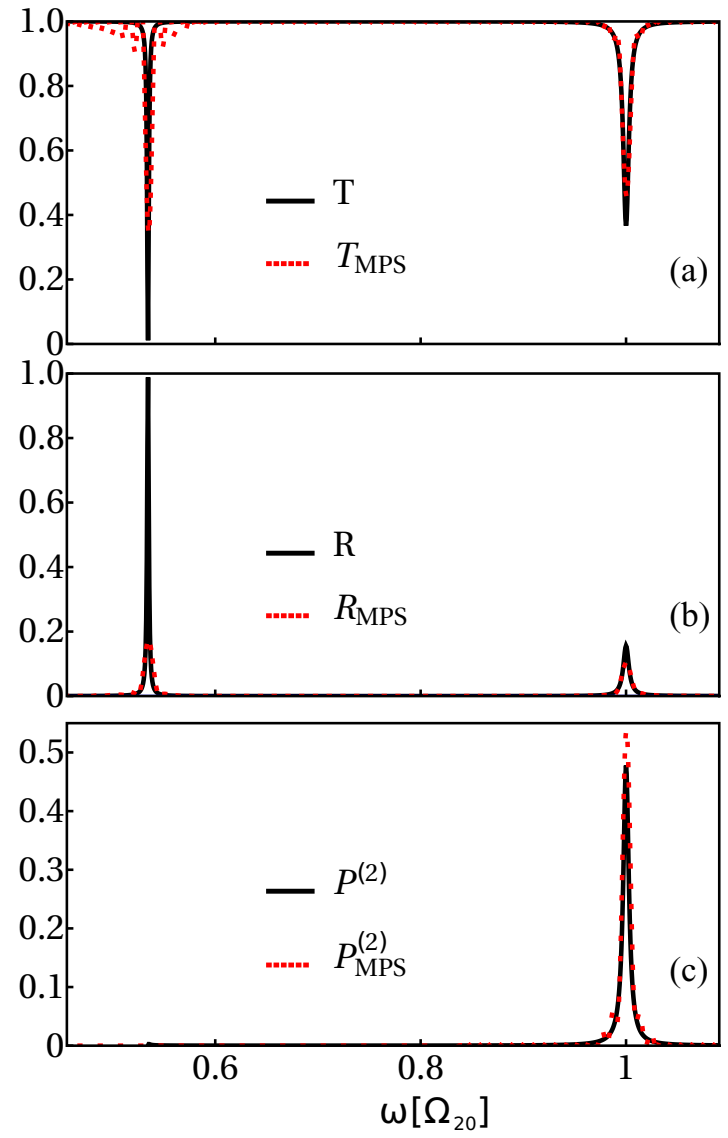

FIG. 4. Scattering coefficients in a cyclic three-level system, as a function of the incident frequency $\omega$. (a) One photon transmittance, (b) reflectance, and (c) probability of two-photon generation $P^{(2)}(\omega)$. We show both analytical (solid lines) and numerical results obtained with MPS (dotted lines). The parameters are $\Omega_{10}=0.53, \Omega_{20}=$ $1, \Gamma_{10}^{(0)}=1 \times 10^{-3}, \Gamma_{20}^{(0)}=1.4 \times 10^{-3}$, and $\Gamma_{21}^{(0)}=2.2 \times 10^{-3}$. We remind that $\Gamma_{i j}^{(0)}=2 \pi D^{2}\left(\Omega_{i j}\right) G_{i j}^{2}$.

given by

$$
P^{(2)}(\omega):=\lim _{t \rightarrow \infty} \frac{\left|\sum_{s, s^{\prime}=r, l} \sum_{\omega_{1} \omega_{2}}^{\prime}\left\langle\Omega\left|s_{\omega_{1}} s_{\omega_{2}}^{\prime} e^{-i H t}\right| \Psi_{\text {in }}\right\rangle\right|^{2}}{2\left|\left\langle\Omega\left|r_{\omega}\right| \psi_{\text {in }}\right\rangle\right|^{2}},
$$

with the summation $\sum_{\omega_{1} \omega_{2}}^{\prime}$ performed over the values of $\omega_{1}$ and $\omega_{2}$ such that they fulfill energy conservation: $\omega_{1}+\omega_{2}=\omega$. In all these expressions, $|\Omega\rangle$ is the ground state of Eq. (1). The first transmission dip occurs when the photon energy is centered around $\omega=\Omega_{10}$. In this spectral region the $|0\rangle \rightarrow|1\rangle$ transition is the only one available. Thus, the C3LS behaves as an effective two-level system and the photon is fully reflected at resonance [13-15]. Consequently, $P^{(2)}(\omega)=0$ in this frequency range [cf. Fig. 4(c)]. In the second transmission dip, located at $\omega=\Omega_{20}$, the transmittance presents a finite minimum value that is close to 0.5 . Figures 4 (b) and 4(c) show a remarkable $50 \%$ down-conversion efficiency of the incoming photon into just two (and only two) outgoing photons, with only a very small amount of light being back-reflected.

For the sake of completeness and to emphasize the fact that we have access to the time domain too, we plot the C3LS level 

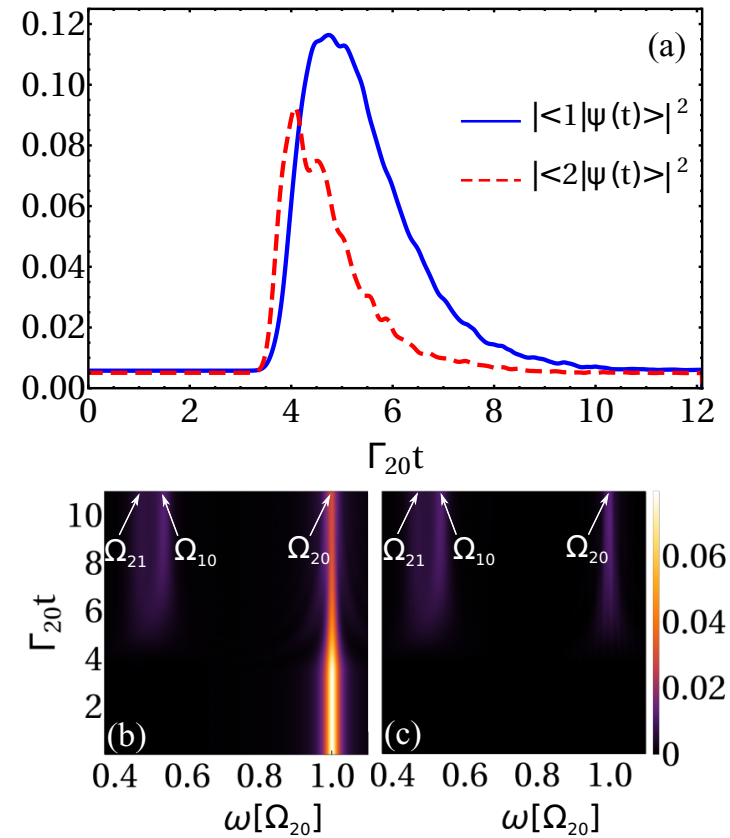

FIG. 5. (a) Population of the first (blue solid line) and second (red dashed line) excited states as a function of time. Photon occupation in energy space for (b) right-moving, $\left\langle n_{\omega}^{(\mathrm{r})}\right\rangle$, and (c) left-moving photons, $\left\langle n_{\omega}^{(\mathrm{l})}\right\rangle$, respectively, as a function of time. The energies are those of Fig. 4. Just to improve the visibility of the figure, we take larger values for the decay rates: $\Gamma_{10}^{(0)}=0.021, \Gamma_{20}^{(0)}=0.028$, and $\Gamma_{21}^{(0)}=0.043$.

population in Fig. 5(a). We see that the second excited state gets populated first, since our incident photon is resonant with the transition $|0\rangle \leftrightarrow|2\rangle$. After the transient period, both levels decay to the ground state. We also plot the particles in energy space, $\left\langle n_{\omega}^{(r)}\right\rangle=\left\langle r_{\omega}^{\dagger} r_{\omega}\right\rangle$ and $\left\langle n_{\omega}^{(l)}\right\rangle=\left\langle l_{\omega}^{\dagger} l_{\omega}\right\rangle$ in Figs. 5(b) and 5(c), respectively. In doing so, we can visualize the two-photon generation in time domain. At the beginning, we have a single peak around the incident energy for the right-moving photons. After the interaction has occurred, a peak has appeared for a left-moving photon at $\Omega_{20}$, corresponding to the single-photon reflection [see Fig. 5(c)]. In addition, two peaks emerge after the scattering for both forward- and backward-traveling photons centered at $\Omega_{21}$ and $\Omega_{10}$, associated to the generation of the two-photon state.

\section{B. Characterization for the two-photon output}

In order to characterize the two-photon wave function emerging from the down-conversion process, we compute the two-point wave function, both in position space $\phi_{x_{1} x_{2}}^{\text {out }}:=$ $\left\langle\Omega\left|a_{x_{1}} a_{x_{2}}\right| \Psi\left(t_{\text {out }}\right)\right\rangle$, where $a_{x}$ annihilates a photon at $x, a_{x}:=$ $1 / \sqrt{2 \pi}\left(\int_{\omega>0} d \omega r_{\omega} e^{i \omega x}+\int_{\omega<0} d \omega l_{\omega} e^{i \omega x}\right)$, and in energy space for right-moving photons $\tilde{\phi}_{\omega_{1} \omega_{2}}^{\text {out }}:=\left\langle\Omega\left|r_{\omega_{1}} r_{\omega_{2}}\right| \Psi\left(t_{\text {out }}\right)\right\rangle$. As shown in Fig. 6, both photons are emitted spatially in a symmetric way with respect to the position of the scatterer $(x=0)$. In energy space, $\tilde{\phi}_{\omega_{1} \omega_{2}}^{\text {out }}$ is centered around $\left(\omega_{1}, \omega_{2}\right)=$ $\left(\Omega_{10}, \Omega_{21}\right)$ and $\left(\Omega_{21}, \Omega_{10}\right)$ (white dotted lines), as expected from emission from a doubly resonant process. However, and similarly to the phenomena of resonance fluorescence, $\tilde{\phi}_{\omega_{1} \omega_{2}}^{\text {out }}$ is
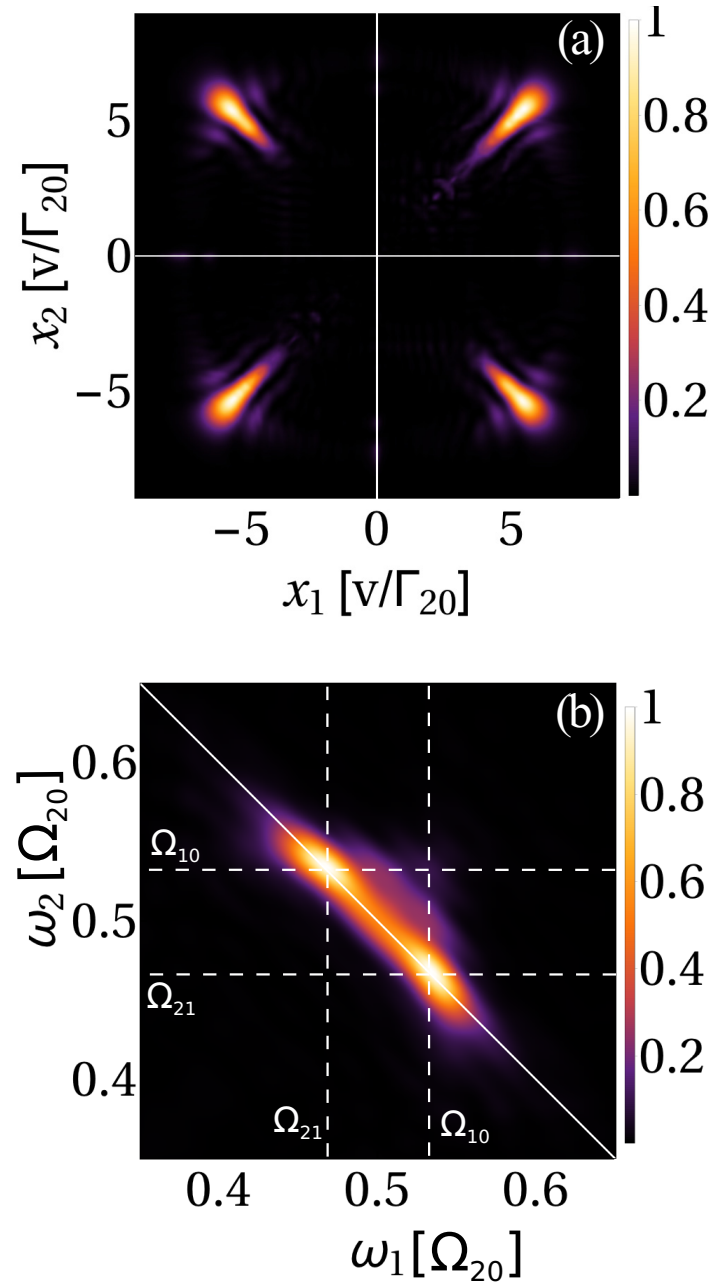

FIG. 6. Square modulus of the two-photon wave function both in (a) position, $\left|\phi_{x_{1} x_{2}}^{\text {out }}\right|^{2}$, and (b) energy space, $\left|\tilde{\phi}_{\omega_{1} \omega_{2}}^{\text {out }}\right|^{2}$. The isoenergetic line, $\omega_{1}+\omega_{2}=\omega$, is shown in the bottom panel (white line). We normalize both wave functions such that $\max \left(\left|\phi_{x_{1} x_{2}}^{\text {out }}\right|^{2}\right)=$ $\max \left(\left|\tilde{\phi}_{\omega_{1} \omega_{2}}^{\text {out }}\right|^{2}\right)=1$. Same parameters as in Fig. 5 .

nonzero all along the isoenergetic curve $\omega_{1}+\omega_{2}=\omega$ [white solid line in Fig. 6(b)].

The emitted photons are entangled. The corresponding von Neumann entropy $S_{\mathrm{VN}}$ can be computed after normalizing the two-photon wave function, such that $\sum_{x_{1} x_{2}}\left|\phi_{x_{1} x_{2}}^{\text {out }}\right|^{2}=1$, and finding its Schmidt decomposition, $\phi_{x_{1} x_{2}}^{\text {out }}=\sum_{m} \lambda_{m} \varphi_{x_{1}, m} \chi_{x_{2}, m}$, being $\left\{\lambda_{m}\right\}$ the singular values. Then $S_{\mathrm{VN}}=-\sum_{m} \lambda_{m}^{2} \ln \left(\lambda_{m}^{2}\right)$ [38]. In the representative case shown in Fig. 6 we get $S_{\mathrm{VN}}=$ 1.44. For a better understanding, we plot the contribution of each mode to $S_{\mathrm{VN}}$ in Fig. 7(a). The entropy is dominated by the first two singular values, but the contribution from the other Schmidt modes is non-negligible. In order to quantify how the entropy is recovered from a given number of singular values, we define the entanglement entropy of the first $m$ Schmidt modes,

$$
S_{\mathrm{VN}, m}:=-\sum_{n=1}^{m} \lambda_{n}^{2} \ln \left(\lambda_{n}^{2}\right),
$$

and show $S_{\mathrm{VN}, m} / S_{\mathrm{VN}}$ in the inset of Fig. 7. 

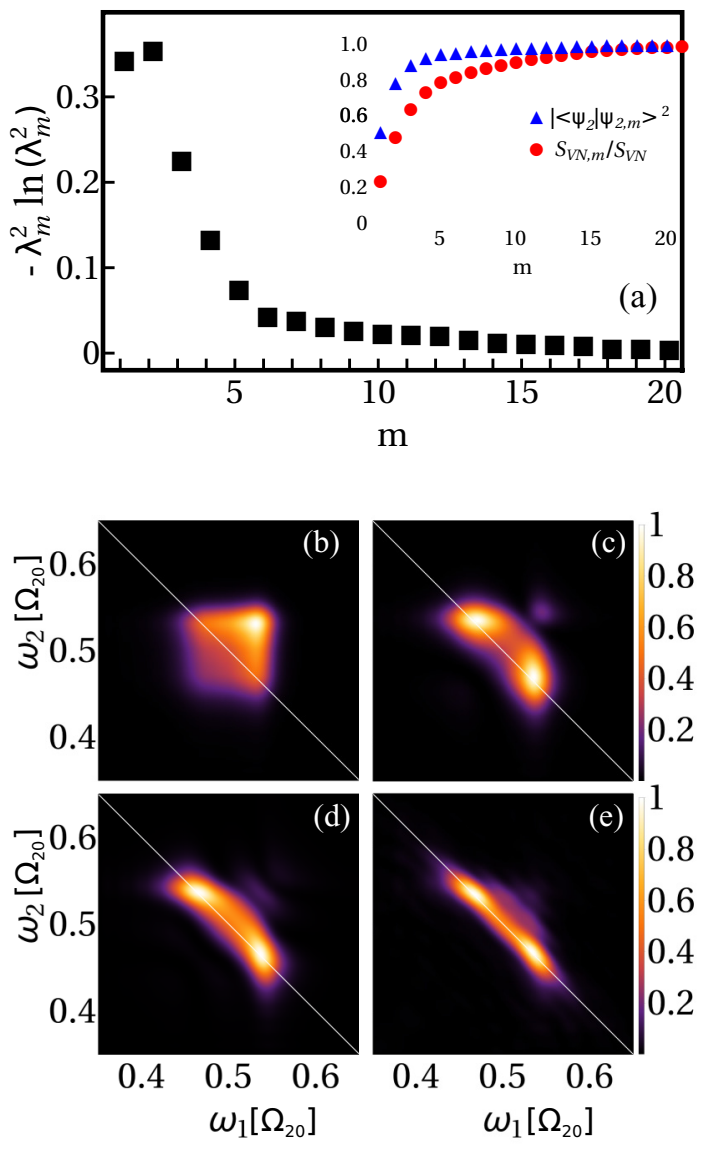

FIG. 7. (a) Contribution of each Schmidt mode to $S_{\mathrm{VN}}$, $-\lambda_{m}^{2} \ln \left(\lambda_{m}^{2}\right)$ as a function of $m$. In the inset, we plot the entropy of $\left|\Psi_{2, m}\right\rangle$ over the whole entropy, $S_{\mathrm{VN}, m} / S_{\mathrm{VN}}$ (red circles), and the overlap between $\left|\Psi_{2}\right\rangle$ and $\left|\Psi_{2, m}\right\rangle$ (blue triangles) as a function of $m$. $\left|\tilde{\phi}_{\omega_{1}, \omega_{2}}^{\text {out, }}\right|^{2}$ for (b) $m=1$, (c) $m=2$, (d) $m=3$, and (e) $m=L$ which is the exact result [cf. Fig. 6(b)]. $L$ is the number of modes, which matches with the number of points in the discretized waveguide; see Appendix D. Same parameters as in Fig. 5.

Another measure of how the wave function can be represented by a fixed number of modes is the fidelity, i.e., the overlap between the actual two-photon state, $\left|\Psi_{2}\right\rangle=$ $1 / \sqrt{2} \sum_{x_{1}, x_{2}} \phi_{x_{1}, x_{2}}^{\text {out }} a_{x_{1}}^{\dagger} a_{x_{2}}^{\dagger}|\Omega\rangle$ and the state reconstructed with $m$ modes:

$$
\left|\Psi_{2, m}\right\rangle:=\frac{1}{\sqrt{2}} \sum_{x_{1}, x_{2}} \phi_{x_{1}, x_{2}}^{\text {out } \mathrm{m}} a_{x_{1}}^{\dagger} a_{x_{2}}^{\dagger}|\Omega\rangle,
$$

where $\phi_{x_{1}, x_{2}}^{\text {out,m }}$ is the two-photon wave function reconstructed with the first $m$ Schmidt modes, $\phi_{x_{1}, x_{2}}^{\text {out,m }}=\sum_{n=1}^{m} \lambda_{n} \tilde{\varphi}_{x_{1}, n} \tilde{\chi}_{x_{2}, n}$. In Fig. 7(a) (inset) we check that the overlap qualitatively behaves as $S_{\mathrm{VN}, \mathrm{m}}$.

Lastly, we can visualize how the two-photon wave function is reconstructed by adding modes. In Figs. 7(b)-7(d), we plot $\left|\tilde{\phi}_{\omega_{1}, \omega_{2}}^{\text {out }}\right|^{2}$ for $m=1,2$, and 3, respectively, whereas we plot $\left|\tilde{\phi}_{\omega_{1}, \omega_{2}}^{\text {out }}\right|$ in Fig. 7(e). The white lines, as in Fig. 6, mark the isoenergetic condition. While the wave-function reconstruction with $m=1$ does not reproduce the bimodal aspect of the state, already with $m=2$ the double-peaked structure is well defined.
We have not discussed how to optimize the two-photon entanglement. Since the two-photon wave packet is created in the $2 \rightarrow 1 \rightarrow 0$ transition we can benefit from the studies on spontaneous emission in cascade systems and argue, as explained in Ref. [39], that maximally entangled states are expected in the regime $\Gamma_{21} / \Gamma_{10} \rightarrow 0$. In our model, this ratio is fixed by the relation $E_{C} / E_{J}$. However, another C3LS or further engineering in the transmon can tune this ratio and provide a way of generating a different amount of entanglement. If our proposal is tried in the laboratory, this point must be tackled in the future.

\section{ANALYTICAL THEORY}

Model (1) is not analytically solvable. However, under fair assumptions we are able to provide rather general expressions for the down-conversion efficiency. First, we remind that we have numerically tested that no more than two photons are generated in the dynamics. Therefore, the two-photon generation probability can be computed by energy conservation as

$$
P^{(2)}(\omega)=1-\left|t^{(1)}(\omega)\right|^{2}-\left|r^{(1)}(\omega)\right|^{2}-A(\omega) .
$$

Here, $t^{(1)}(\omega)$ and $r^{(1)}(\omega)$ are the one-photon transmittance and reflection, respectively. The last term is the energy "absorbed" by the lossy channels, $A(\omega)$. Besides, the C3LS is assumed punctual. Thus, the photonic wave function is continuous in the scatterer position, implying $r^{(1)}(\omega)=t^{(1)}(\omega)-1$ [40].

We first summarize the calculation of the single-photon transmittance. Here, we provide the main results, sending the full derivation to Appendix A. It turns out that $t^{(1)}(\omega)$ can be calculated as

$$
t^{(1)}(\omega)=\lim _{\alpha \rightarrow 0} \frac{\left\langle\alpha_{\omega}\left|r_{\text {out }}(t)\right| \alpha_{\omega}\right\rangle}{\left\langle\alpha_{\omega}\left|r_{\text {in }}(t)\right| \alpha_{\omega}\right\rangle}
$$

with the input and output fields [41] $r_{\text {in }}(t):=$ $\int_{0}^{\infty} \frac{d \omega}{\sqrt{2 \pi}} r_{\omega}\left(t_{0}\right) e^{-i \omega\left(t-t_{0}\right)}$ and $r_{\text {out }}(t):=\int_{0}^{\infty} \frac{d \omega}{\sqrt{2 \pi}} r_{\omega}\left(t_{f}\right) e^{-i \omega\left(t-t_{f}\right)}$ with $r_{\omega}(t)=e^{i H t} r_{\omega} e^{-i H t}$. The times $t_{0}$ and $t_{f}$ must be taken well before and after the scattering event has occurred. As we are interested in asymptotic behavior, we can set $t_{0} \rightarrow-\infty$ and $t_{f} \rightarrow \infty$. Besides, $\left|\alpha_{\omega}\right\rangle=e^{\alpha r_{\text {in }}^{\dagger}(\omega)-\text { H.c. }}|\Omega\rangle$, where $r_{\text {in }}^{\dagger}(\omega)$ is the Fourier transform of $r_{\text {in }}^{\dagger}(t)$. Thus, the one-photon transmission (reflection) can be obtained by sending a coherent state through the scatterer and computing the dynamics for the fields up to first order in the input amplitude $\alpha$.

The usefulness of Eq. (13) comes with our second result,

$$
\begin{aligned}
& \lim _{\alpha \rightarrow 0}\left\langle\alpha_{\omega}\left|r_{\text {out }}(t)\right| \alpha_{\omega}\right\rangle \\
& \quad=\lim _{\alpha \rightarrow 0}\left\langle\alpha_{\omega}\left|r_{\text {in }}(t)\right| \alpha_{\omega}\right\rangle-i \sqrt{2 \pi} D(\omega) \alpha G(\omega) e^{-i \omega t},
\end{aligned}
$$

where $G(\omega)$ is the linear susceptibility for $G(t)$ when a classical drive $H_{\text {drive }}=2 \alpha \cos (\omega t) G$ is added to Eq. (1). Summarizing, the single-photon transmittance can be calculated by means of the C3LS response to a classical drive. This problem is still hard since the total model, Eq. (1), is a many-body Hamiltonian. We need to do an approximation. We consider that the line-C3LS coupling strength can be considered up to second order. In doing so, the scatterer dynamics is given by the quantum optical master equation [41] for the reduced 
density matrix of the C3LS:

$$
\begin{aligned}
\frac{d \varrho}{d t}= & -i\left[\sum_{j=0}^{2} \Omega_{j}|j\rangle\langle j|, \varrho\right]-i \alpha D(\omega) 2 \cos (\omega t)[G(t), \varrho] \\
& +2 \sum_{\Omega_{i j}>0} \Gamma_{i j}\left(L_{i j} \varrho L_{i j}^{\dagger}-\frac{1}{2}\left\{L_{i j}^{\dagger} L_{i j}, \varrho\right\}\right) \\
& +\mathcal{D} \varrho \mathcal{D}-\frac{1}{2}\left\{\mathcal{D}^{2}, \varrho\right\} .
\end{aligned}
$$

The first term is the C3LS Hamiltonian contribution. The second stands for the classical driving discussed above. The second line contains the Lindblad terms describing both the hopping to the line and dissipative channels. There, $L_{i j}=$ $|j\rangle\langle i|$ and $\Gamma_{i j}$ are the transition rates between the discrete levels in the scatterer. In addition to the transition rates induced by coupling to the waveguide photons this formulation allows us to consider the transitions $\gamma_{i j}$ induced by coupling to other baths (as phonons or other components of the EM field). In this case, the total transition rate is $\Gamma_{i j}=\Gamma_{i j}^{(0)}+\gamma_{i j}$. The third line stands for pure dephasing, with $\mathcal{D}$ being diagonal matrices. The dephasing rates simply modify the nonradiative losses $\gamma_{i j}$ in the calculation for $t^{(1)}(\omega)$.

The calculation is now possible. After some algebra, we get (cf. Appendix A)

$$
\begin{aligned}
t^{(1)}(\omega)= & 1-\frac{i \Gamma_{10}^{(0)}}{\left(\omega-\Omega_{10}\right)+i\left(\Gamma_{10}+\Gamma_{10}^{\phi}\right)} \\
& -\frac{i \Gamma_{20}^{(0)}}{\left(\omega-\Omega_{20}\right)+i\left(\Gamma_{21}+\Gamma_{20}+\Gamma_{20}^{\phi}\right)},
\end{aligned}
$$

with $\Gamma_{i j}^{\phi}=\frac{1}{2}\left(\mathcal{D}_{i i}-\mathcal{D}_{j j}\right)^{2}$ being the dephasing rates for the $\varrho_{i j}$ elements. Finally, the energy that does not end up in the waveguide can be approximated by (see also Appendix A)

$$
A\left(\omega \cong \Omega_{20}\right)=2 \gamma_{20}\left|r^{(1)}\left(\Omega_{20}\right)\right|^{2} / \Gamma_{20} .
$$

The validity of this analytical formalism is shown in Fig. 4, where it is compared to the numerical results for the "lossless" case $\gamma_{i j}=0$.

Some final comments may be relevant. In the presented theory, the main approximation is to assume that the C3LS-line coupling strength is sufficiently small. This allows the use of the master equation (15) for computing the scatterer dynamics. This is a good assumption in (almost) every experiment so far. Apart from this approximation the theory is rather general, independent of the actual values for dephasing, nonradiative losses or spectral function for the waveguide.

\section{EFFICIENCY}

Equation (12) allows for the search of optimal parameters for down-conversion. The first observation is that losses are detrimental, always reducing $P^{(2)}(\omega)$. Even in the absence of losses $\left(\gamma_{i j}=0\right)$, the two-photon generation can be considered as a loss mechanism for the one-photon channel, which implies that the fraction of energy down-converted is at most $\max P^{(2)}(\omega)=\frac{1}{2}$ [occurring when $r(\omega)=-\frac{1}{2}$ ]. This fundamental bound is related to the fact that, for a deep

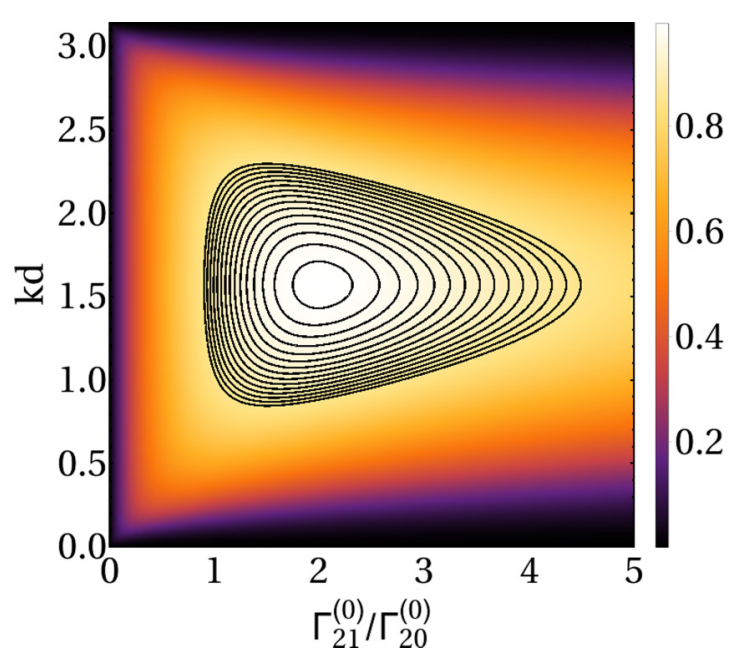

FIG. 8. $P^{(2)}\left(\omega=\Omega_{20}\right)$ as a function of the atom-mirror distance $k d$ (see Fig. 1) and the ratio $\Gamma_{12}^{(0)} / \Gamma_{02}^{(0)}$. Losses are taken into account. In the figure, a conservative ratio $\left(\gamma_{20}+\Gamma_{20}^{\phi}\right) / \Gamma_{20}^{(0)}=0.01$ is used. The rest of the parameters are the same as in Fig. 4. Black lines mark isoefficiency curves, starting at 0.85 and finishing at 0.98 .

subwavelength scatterer emitting equally to the left and to the right, as we have already mentioned, transmittance and reflection are related: $r^{(1)}(\omega)=t^{(1)}(\omega)-1$. Therefore, in the absence of losses, Eq. (12) depends just on $t^{(1)}(\omega)$ and can be maximized, giving the aforementioned bound [42]. By simple inspection of Eq. (16) we see that happens when $\Gamma_{21}^{(0)}=\Gamma_{20}^{(0)}$. But this bound can be exceeded by breaking the left-right symmetry in the waveguide by, e.g., placing a mirror next to the C3LS, as sketched in Fig. 1(b).

Both reflectance and (for $\gamma_{i j} \neq 0$ ) absorption can be calculated in this configuration by summing all multiple-scattering processes that the waveguide photon has with both the C3LS and the mirror [43]. The sum can be done analytically (see Appendix C), resulting in

$$
\begin{aligned}
P^{(2)}(\omega)= & 1-\left|\frac{r^{(1)}(\omega)-\left(1+2 r^{(1)}(\omega)\right) \Phi(\omega)}{1+r^{(1)}(\omega) \Phi(\omega)}\right|^{2} \\
& -\left|\frac{1-\Phi(\omega)}{1+r^{(1)}(\omega) \Phi(\omega)}\right|^{2} A(\omega),
\end{aligned}
$$

where $\Phi(\omega)=e^{2 i k(\omega) d}, d$ is the distance between the mirror and the C3LS, Fig. 1(b), and $k(\omega)$ is the waveguide photon wave vector at frequency $\omega$.

As drawn in Fig. 8, the maximum down-conversion efficiency predicted by Eq. (18) occurs at resonance $\left(\omega=\Omega_{20}\right)$, and for $\Gamma_{21}^{(0)} / \Gamma_{20}^{(0)} \cong 2$ and $k d=\pi / 2$, and can be approximated by

$$
\max P_{2}=1-\frac{\gamma_{20}+\Gamma_{20}^{\phi}}{\Gamma_{20}} .
$$

So, remarkably, down-conversion may be perfect in the considered configuration if losses are negligible. Equation (19) provides a simple expression for the maximum efficiency as a function of the ratio between the rates for absorption and coupling into waveguide photons. This ratio is a key figure of merit in waveguide QED and values as small as $1 \times 10^{-2}$ have 
already been reported for effective two-level systems in both superconducting circuits [8] and photonic crystals [44]. Thus, two-photon generation with one and only one photon with an efficiency larger than 0.99 is doable using an appropriate C3LS.

\section{CONCLUSIONS}

We have shown that two photons can be efficiently generated by sending one and only one photon through a cyclic three-level atom in a realistic scenario. Remarkably, the down-conversion process can occur with unit probability, being only limited by energy leakage to other continua rather than the line and dephasing in the three-level system. Based on reported experimental data, we have estimated that a nearly perfect two-photon generator operating at the single-photon level is feasible in architectures based on either photonic crystals or superconducting circuits. Together with single atomic mirrors [13-15], single-photon lasing [16], or single-photon Raman scattering [19], this work contributes to the toolbox of photonics with minimum power, where tasks usually associated to high intensities are performed at the one-photon level.

\section{ACKNOWLEDGMENTS}

We acknowledge support by the Spanish Ministerio de Economia y Competitividad within Projects No. MAT201453432-C5-1-R, No. FIS2012-33022, and No. FIS2014-55867$\mathrm{P}$, the CAM Research Network QUITEMAD+, and the Gobierno de Aragón (FENOL group).

\section{APPENDIX A: ONE-PHOTON SCATTERING, INPUT-OUTPUT, AND LINEAR RESPONSE THEORY}

The one-photon transmittance can be written as $[45,46]$

$$
t^{(1)}(\omega)=\lim _{t \rightarrow \infty} \frac{\left\langle\Omega\left|r_{\omega} r_{\mathrm{out}}^{\dagger}(t)\right| \psi_{\text {in }}\right\rangle}{\left\langle\Omega\left|r_{\omega} r_{\text {in }}^{\dagger}(t)\right| \psi_{\text {in }}\right\rangle},
$$

with $|\Omega\rangle$ the ground state of Eq. (1), $\left|\psi_{\text {in }}\right\rangle$ the initial singlephoton wave packet, and the input-output fields defined in [41]:

$$
\begin{aligned}
r_{\mathrm{in}}(t) & :=\int_{0}^{\infty} \frac{d \omega}{\sqrt{2 \pi}} r_{\omega}\left(t_{0}\right) e^{-i \omega\left(t-t_{0}\right)} \\
r_{\mathrm{out}}(t) & :=\int_{0}^{\infty} \frac{d \omega}{\sqrt{2 \pi}} r_{\omega}\left(t_{f}\right) e^{-i \omega\left(t-t_{f}\right)}
\end{aligned}
$$

Here, $r_{\omega}(t)=e^{i H t} r_{\omega} e^{-i H t}$ are Heisenberg evolved operators. The times $t_{0}$ and $t_{f}$ are times well before, and well after, the scatterer and the incident photons have interacted, respectively. If we are interested in asymptotics, we can set $t_{0} \rightarrow-\infty$ and $t_{f} \rightarrow \infty$. Below, we find an alternative formula for $t^{(1)}(\omega)$.

Instead of a single-photon wave packet, we now consider a coherent input state,

$$
\left|\alpha_{\omega}\right\rangle=e^{\alpha r_{\text {in }}^{\dagger}(\omega)-\text { H.c. }}|\Omega\rangle,
$$

and we compute the expected value,

$$
f_{\text {out }}\left(\omega, \omega^{\prime}, \alpha\right):=\left\langle\alpha_{\omega}\left|r_{\text {out }}\left(\omega^{\prime}\right)\right| \alpha_{\omega}\right\rangle,
$$

with $r_{\text {out }}(\omega)$ being the Fourier transform of $r_{\text {out }}(t)$. Then, we take a series expansion in $\alpha$,

$$
f_{\text {out }}\left(\omega, \omega^{\prime}, \alpha\right)=\alpha\left\langle\Omega\left|r_{\text {out }}\left(\omega^{\prime}\right) r_{\text {in }}^{\dagger}(\omega)\right| \Omega\right\rangle+O\left(\alpha^{2}\right),
$$

that, together with $\left\langle\Omega\left|r_{\text {out }}\left(\omega^{\prime}\right) r_{\text {in }}^{\dagger}(\omega)\right| \Omega\right\rangle=t^{(1)}(\omega) \delta\left(\omega-\omega^{\prime}\right)$, establishes the relation

$$
f_{\text {out }}\left(\omega, \omega^{\prime}, \alpha\right)=\alpha t^{(1)}(\omega) \delta\left(\omega-\omega^{\prime}\right)+O\left(\alpha^{2}\right) .
$$

Fourier transforming with respect to $\omega^{\prime}$,

$$
\begin{aligned}
f_{\text {out }}(\omega, t, \alpha) & :=\frac{1}{\sqrt{2 \pi}} \int d \omega^{\prime} f_{\text {out }}\left(\omega, \omega^{\prime}, \alpha\right) e^{i \omega^{\prime} t} \\
& =\frac{\alpha}{\sqrt{2 \pi}} t^{(1)}(\omega) e^{i \omega t}+O\left(\alpha^{2}\right),
\end{aligned}
$$

where $f_{\text {out }}(\omega, t, \alpha)=\left\langle\alpha_{\omega}\left|r_{\text {out }}(t)\right| \alpha_{\omega}\right\rangle$. Taking all together, the desired equivalent way of computing the single-photon transmittance, Eq. (13), is obtained.

\section{APPENDIX B: LINEAR RESPONSE THEORY AND INPUT-OUTPUT} [41]:

The input and output fields, Eqs. (A2) and (A3), are related

$$
r_{\text {out }}(t)=r_{\text {in }}(t)-i \int_{0}^{\infty} \frac{d \omega^{\prime}}{\sqrt{2 \pi}} \int_{t_{0}}^{t_{f}} d \tau D\left(\omega^{\prime}\right) e^{-i \omega^{\prime}(t-\tau)} G(\tau) .
$$

This general relation can be simplified if we are interested in the single-photon transmittance. As shown above, $t^{(1)}(\omega)$ in Eq. (13) can be computed using a classical driving (a coherent state) in the limit of zero amplitude. Thus, we can use linear response theory to compute $G$ :

$$
\langle G(\tau)\rangle=\alpha G(\omega) e^{i \omega \tau}+\text { c.c. }
$$

where $G(\omega)$ is the linear susceptibility and $\omega$ the frequency of the input state; see Eq. (A4). Replacing the latter in Eq. (B1), we get

$$
\begin{aligned}
\lim _{\alpha \rightarrow 0}\left\langle r_{\text {out }}(t)\right\rangle= & \lim _{\alpha \rightarrow 0}\left\langle r_{\text {in }}(t)\right\rangle-i \int_{0}^{\infty} \frac{d \omega^{\prime}}{\sqrt{2 \pi}} e^{-i \omega^{\prime} t} D\left(\omega^{\prime}\right) \\
& \times \int_{-\infty}^{\infty} d \tau\left(e^{+i\left(\omega^{\prime}-\omega\right) \tau} G\left(\omega^{\prime}\right)+e^{+i\left(\omega^{\prime}+\omega\right) \tau} G^{*}\left(\omega^{\prime}\right)\right) \\
= & \left\langle r_{\text {in }}(t)\right\rangle-i \sqrt{2 \pi} D(\omega) \alpha G(\omega) e^{-i \omega t},
\end{aligned}
$$

which is a remarkable result, telling that the single-photon transmittance can be obtained by means of computing the linear response of $G$.

\section{Calculation of the susceptibility}

In order to compute $G(\omega)$ we split the master equation (15) as

$$
\partial \varrho=\mathcal{L}_{0} \varrho+\alpha 2 \cos (\omega t) \mathcal{L}_{1} \varrho
$$


with the unperturbed

$$
\begin{aligned}
\mathcal{L}_{0}= & -i\left[\sum_{j=0}^{2} \Omega_{j}|j\rangle\langle j|, \varrho\right] \\
& +2 \sum_{\Omega_{i j}>0} \Gamma_{i j}\left(L_{i j} \varrho L_{i j}^{\dagger}-\frac{1}{2}\left\{L_{i j}^{\dagger} L_{i j}, \varrho\right\}\right) \\
& +\mathcal{D} \varrho \mathcal{D}-\frac{1}{2}\left\{\mathcal{D}^{2}, \varrho\right\},
\end{aligned}
$$

and perturbed part

$$
\mathcal{L}_{1} \varrho=-i D(\omega)[G(t), \varrho] .
$$

In the absence of perturbation $\mathcal{L}_{1}=0$ the solution of the master equation can be written as (in the C3LS eigenbasis)

$$
\varrho_{i j}(t)=\varrho_{i j}(0) e^{-\left(i \Omega_{i j}+\kappa_{i j}\right) t}
$$

where $\kappa_{i j}$ is in general a sum of some $\Gamma$ s and the elements of $\mathcal{D}$. With this at hand we can use the linear response theory and write (see Ref. [47], Chap. 6)

$$
\begin{aligned}
G(\omega) & =G_{r}(0)+i \omega \int_{0}^{\infty} d s G_{r}(s) e^{i \omega s} \\
& =\sum_{i j} G_{i j} \rho_{j i}(0)\left(1+i \omega \int_{0}^{\infty} d s e^{-\left[\kappa_{j i}+i\left(\Omega_{j i}-\omega\right)\right] s}\right) \\
& =\sum_{i j} G_{i j} \rho_{j i}(0) \frac{\kappa_{j i}+i \Omega_{j i}}{\kappa_{j i}+i\left(\Omega_{j i}-\omega\right)} \\
& \cong \sum_{\Omega_{j i} \cong \omega} G_{i j} \rho_{j i}(0) \frac{\kappa_{j i}+i \Omega_{j i}}{\kappa_{j i}+i\left(\Omega_{j i}-\omega\right)}
\end{aligned}
$$

Here, $G_{r}(s)$ stands for the unperturbed evolution where the initial conditions are those computed with the stationary solution for the master equation: $\mathcal{L}_{0}+\alpha \mathcal{L}_{1}$, i.e., the total master equation (15) with a constant perturbation (without the cosine modulation). The last approximation considers that the main contribution comes from the terms with frequencies $\Omega_{i j}$ closer to the driven frequency $\omega$.

\section{Transmission calculation}

In formula (B8) we first need to compute the stationary solutions $\varrho_{i j}(0)$ with constant perturbation [see our previous discussion below Eq. (B8)]. Since we are assuming zero temperature, it is clear that $\varrho_{11} \sim \varrho_{22} \sim O(\alpha)$ and $\varrho_{00} \sim$ $1-O(\alpha)$. Taking this into account, the solution for the nondiagonal elements is as follows:

$$
\begin{aligned}
\dot{\varrho}_{10}=0= & -i \Omega_{10} \varrho_{10}-i \alpha G_{10} D(\omega) \\
& -\left(\Gamma_{10}+\frac{1}{2}\left(\mathcal{D}_{11}-\mathcal{D}_{00}\right)^{2}\right) \varrho_{10}+O\left(\alpha^{2}\right), \\
\dot{\varrho}_{20}=0= & -i \Omega_{20} \varrho_{20}-i \alpha G_{20} D(\omega) \\
& -\left(\Gamma_{20}+\Gamma_{21}+\frac{1}{2}\left(\mathcal{D}_{22}-\mathcal{D}_{00}\right)^{2}\right) \varrho_{20}+O\left(\alpha^{2}\right) .
\end{aligned}
$$

Solving these equations and inserting them in the general expression (B8) together with relation (14) yields Eq. (16) in the main text.

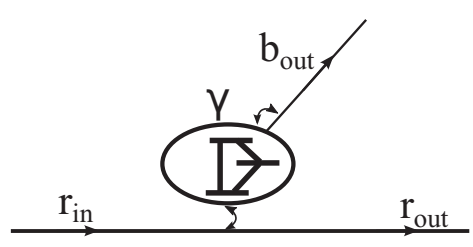

FIG. 9. Schematics for the modeling of nonradiative losses.

\section{Leakage}

Losses can be modeled as decays to other channels, which are characterized by input and output fields named here as $b_{\text {in }}$ and $b_{\text {out }}$. Here, we take into account one additional channel (others will simply sum); see Fig. 9. The input-output relations (B3) must be generalized now to include this extra channel,

$$
\begin{gathered}
r_{\mathrm{out}}(t)=r_{\mathrm{in}}(t)-i \sqrt{2 \pi} D(\omega) G(t), \\
b_{\mathrm{out}}(t)=b_{\mathrm{in}}(t)-i \sqrt{2 \gamma} G(t) .
\end{gathered}
$$

The quantity $\gamma$ parametrizes a phenomenological loss rate, and the 2 in front is because we do not consider left and right modes in the nonradiative channel but just $b$ modes. Besides, $b_{\text {in }}(t)=0$ and the transmission in the $b$ modes reads

$$
\tau(\omega)=\frac{-i \sqrt{2 \gamma}\langle G\rangle}{\left\langle r_{\text {in }}\right\rangle} .
$$

\section{APPENDIX C: EFFICIENCY CALCULATIONS}

In order to compute the reflection and leakage when the mirror is placed, we must sum over all the possible reflection, transmission, and leakage events, as shown in Fig. 10. In doing so, we define $\Phi(\omega)=\exp (i k(\omega) d)$, which is the phase accumulated by a photon with quasimomentum $k$ traveling a distance $d$ (this will be the distance between the mirror and the atom). Finally, we denote the reflection in the mirror as $r_{M}$. Eventually, we set $r_{M}=-1$; i.e., we neglect losses in the mirror, which is a good assumption in many experimental setups.

With the mirror, $P^{(2)}(\omega)$ is written as

$$
P^{(2)}(\omega)=1-\left|r_{\text {tot }, 1}(\omega)\right|^{2}-\left|\tau_{\text {tot }}(\omega)\right|^{2},
$$

where $r_{\text {tot, } 1}(\omega)$ is the total one-photon reflection. It should be distinguished from $r^{(1)}(\omega)$, which stands for the reflection occurring in every event. Finally, $\tau_{\text {tot }}(\omega)$ is the total leakage. Summing over all scattering events (see Fig. 10), we finally get

$$
\begin{aligned}
r_{\mathrm{tot}}^{(1)}(\omega)= & r^{(1)}(\omega)+t^{(1)}(\omega) \Phi(\omega) r_{M} t^{(1)}(\omega) \\
& +t^{(1)}(\omega) \Phi(\omega) r_{M} t^{(1)}(\omega) r^{(1)}(\omega) \Phi(\omega) r_{M} t^{(1)}(\omega) \\
& +\cdots \\
= & r^{(1)}(\omega)+\frac{\left(t^{(1)}(\omega)\right)^{2} \Phi(\omega) r_{M}}{1-r^{(1)}(\omega) \Phi(\omega) r_{M}}
\end{aligned}
$$

and

$$
\begin{aligned}
\tau_{\text {tot }}(\omega)= & \tau(\omega)+t^{(1)}(\omega) \Phi(\omega) r_{M} \tau(\omega) \\
& +t^{(1)}(\omega) \Phi(\omega) r_{M} t^{(1)}(\omega) r^{(1)}(\omega) \Phi(\omega) r_{M} \tau(\omega) \\
& +\cdots \\
= & \tau(\omega)+\frac{\tau(\omega) t^{(1)}(\omega) \Phi(\omega) r_{M}}{1-r^{(1)}(\omega) \Phi(\omega) r_{M}}
\end{aligned}
$$




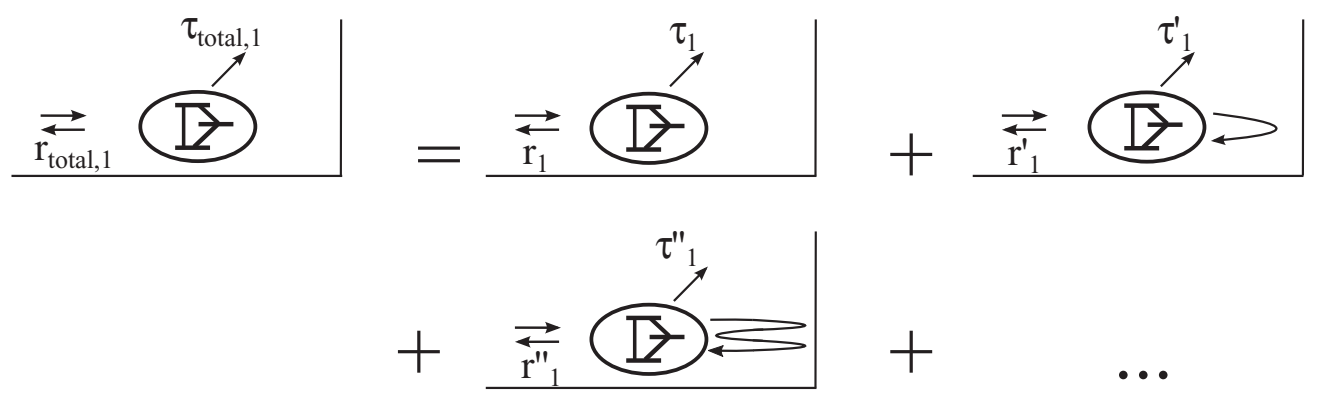

FIG. 10. Diagrammatic representation for the possible scattering events giving the total reflection.

Combining Eqs. (C2) and (C3) with Eq. (C1) we can compute the two-photon generation $P^{(2)}(\omega)$, considering $r_{M}=-1$. The transmission into the auxiliary modes is the energy loss from the system, i.e.,

$$
A(\omega) \equiv|\tau(\omega)|^{2}
$$

\section{APPENDIX D: NUMERICAL SIMULATIONS}

\section{Matrix product states}

We are studying the dynamics of a state with one or two photons flying over the ground state. As this state is expected to have a small amount of entanglement, we can use the variational ansatz of matrix product states $[34,35]$ to describe the discrete wave function for scattering problems as we have shown recently $[19,36]$. This ansatz has the form

$$
|\psi\rangle=\sum_{s_{x} \in\left\{1, d_{x}\right\}} \operatorname{tr}\left[\prod A_{x}^{s_{x}}\right]\left|s_{1}, s_{2}, \ldots, s_{L}\right\rangle .
$$

It is constructed from $L$ sets of complex matrices $A_{x}^{s_{x}} \in$ $M\left[\mathbb{C}^{D}\right]$, with $L$ the number of sites, where each set is labeled by the quantum state $s_{x}$ of the corresponding site. The local Hilbert space dimension $d_{x}$ is infinity, since we are dealing with bosonic sites. During the dynamics, processes that create more than two photons are still highly off resonance. In consequence, we can truncate the bosonic space and consider states with zero to $n_{\max }$ photons per cavity. The composite Hilbert space is $\mathcal{H}=\bigotimes_{x} \mathbb{C}^{d_{x}}$, where the dimension is $d_{x}=n_{\max }+1$ for the empty resonators and $d_{x_{0}}=3\left(n_{\max }+1\right)$ for the cavity with the three-level system. We thus expect the composite wave function of the photon C3LS to consist of a superposition with a small number of photons.

The total number of variational parameters is $(L-$ 1) $D^{2}\left(n_{\max }+1\right)+3 D^{2}\left(n_{\max }+1\right)$ and depends on the size of the matrices, $D$. The key point is that, for describing a general state, $D$ increases exponentially with $L$, whereas it increases only polynomially with $L$ if the entanglement is small enough.

The computation of scattering matrices with MPSs uses four different algorithms. The most basic one is to create product state such as a vacuum state with the deexcited C3LS: $|\psi\rangle=|0\rangle \mid$ vac $\rangle$. These states can be reproduced using matrices of bond dimension $D=1$, so each matrix is just a coefficient $A_{x}^{s_{x}}=\delta_{s_{x}}$. The second algorithm is to compute expectation values from MPSs. This amounts to a contraction of tensors that can be performed efficiently [34] and allows one to compute single-site operators $\left\langle a_{x}^{\dagger} a_{x}\right\rangle,\left\langle\sigma_{z}\right\rangle$, correlators as $\left\langle a_{x}^{\dagger} a_{y}\right\rangle$, or even projections as $\left\langle\Omega\left|a_{x_{1}} a_{x_{2}}\right| \psi\right\rangle$. The third operation we need to perform is to apply operators on a state, $O|\psi\rangle$, such as introducing or removing excitations $a_{x}^{\dagger}|\psi\rangle$. We do this in an efficient fashion by interpreting the operator $O$ as a matrix product operator (MPO) [48]. A MPO is a matrix product representation of an operator:

$$
O=\sum_{s_{x}, s_{x}^{\prime} \in\left\{1, d_{x}\right\}} \operatorname{tr}\left[\prod B_{x}^{s_{x}, s_{x}^{\prime}}\right]\left|s_{1}, s_{2}, \ldots, s_{L}\right\rangle\left\langle s_{1}^{\prime}, s_{2}^{\prime}, \ldots, s_{L}^{\prime}\right| .
$$

So, now we have $L$ sets of complex matrices $B_{x}^{s_{x}, s_{x}^{\prime}} \in M\left[\mathbb{C}^{D_{o}}\right]$, where each set is labeled by two indices $s_{x}, s_{x}^{\prime}$ of the corresponding site.

We just need to apply sums of one-body operators:

$$
O=a_{\phi}^{\dagger}=\sum_{x} \phi_{x} a_{x}^{\dagger}
$$

In such a case, an efficient representation of the MPO is obtained with $D_{O}=2$ :

$$
B_{x}^{s_{x}, s_{x}^{\prime}}=\left(\begin{array}{cc}
\delta_{s_{x}, s_{x}^{\prime}} & 0 \\
\phi_{x}\left(a_{x}^{\dagger}\right)_{s_{x}, s_{x}^{\prime}} & \delta_{s_{x}, s_{x}^{\prime}}
\end{array}\right), \quad x=2,3, \ldots, L-1,
$$

whereas $\quad B_{1}^{s_{1}, s_{1}^{\prime}}=\left(\phi_{1}\left(a_{1}^{\dagger}\right)_{s_{1}, s_{1}^{\prime}}, \delta_{s_{1}, s_{1}^{\prime}}\right) \quad$ and $\quad B_{L}^{s_{L}, s_{L}^{\prime}}=$ $\left(\delta_{s_{L}, s_{L}^{\prime}}, \phi_{L}\left(a_{L}^{\dagger}\right)_{s_{L}, s_{L}^{\prime}}\right)^{T}$, with $\left(a_{x}^{\dagger}\right)_{s_{x}, s_{x}^{\prime}}=:\left\langle s_{x}\left|a_{x}^{\dagger}\right| s_{x}^{\prime}\right\rangle$.

Finally, we can also evaluate the time evolution by repeatedly contracting the state with an MPO approximation of the unitary operator $\exp (-i H \Delta t)$ for short times, and truncating it to an ansatz MPS with a fixed $D$. Since our problem does not contain long-range interactions and since the state is well approximated by MPS, it is sufficient to rely on a third-order Suzuki-Trotter formula [49]. We can also take imaginary time evolution to obtain the ground state by solving the equation $\frac{d}{d t}|\psi\rangle=-H|\psi\rangle$ for finite time steps while constantly normalizing the state. Provided a suitable initial state, the algorithm converges to the lowest-energy state of $H$. Notice that the ground state is totally necessary in order to study the dynamics, since the initial state is obtained by applying a single-body operator as that of Eq. (D3) over the ground state. 


\section{Simulated model: Input state and parameters used}

To simulate the waveguide, we consider a one-dimensional array of coupled cavities:

$$
\begin{aligned}
H= & \epsilon \sum_{x} a_{x}^{\dagger} a_{x}-J \sum_{x}\left(a_{x}^{\dagger} a_{x+1}+\text { H.c. }\right) \\
& +\sum_{i} \omega_{i}|i\rangle\langle i|+\sum_{i j}\left(G_{i j}|i\rangle\langle j|+\text { H.c. }\right)\left(a_{0}+a_{0}^{\dagger}\right)
\end{aligned}
$$

where $\epsilon$ are the bare frequencies of the cavities, $J$ is the hopping between nearest neighbors, and $G_{i j}$ is the coupling constant for the $|i\rangle \leftrightarrow|j\rangle$ transition. The lattice spacing $d$ is fixed to 1 . The photonic part can be diagonalized in momentum space, giving the dispersion relation $\omega(k)=$ $\epsilon-2 J \cos k$. The density of electromagnetic modes will be $D(\omega)=1 / \sqrt{2 J|\sin (k(\omega))|}$.

We fix $\epsilon=1, J=1 / \pi, \omega_{0}=0, \omega_{1}=0.59$, and $\omega_{2}=1.10$ (these energies were obtained from the model introduced in the main part of the text). We take $L=1000$ cavities and we place the scatterer at the center, which we define as $x_{0}=0$. The couplings used in the simulations to compute the full spectrum are $G_{01}=-0.0225$, and $G_{12}=G_{02}=0.03$. In the simulations in which we computed the two-photon wave function, in order to get a cleaner scattering state and due to limitations in the time of simulation, we artificially increased the couplings: $G_{01}=-0.10, G_{12}=G_{02}=0.13$.

We work in position space. The input state is

$$
\left|\Psi_{\text {in }}\right\rangle=\sum_{x} e^{i k_{0} x} e^{(x-\bar{x}) / 2 \sigma} \theta(\bar{x}-x) a_{x}^{\dagger}|\Omega\rangle,
$$

up to a normalization constant, with $\bar{x}$ the position of the wave front, $\sigma$ the width, $k_{0}$ the mean momentum, and $\theta(x)$ the Heaviside function. We fix $\bar{x}=420$ and $k_{0}=1.73$ (on resonance with $\Omega_{20}$ ). We take $\sigma=2$ for the simulations to get the full spectrum and $\sigma=20$ for the simulation in which we compute dynamical properties, Fig. 5, and the two-photon wave function [Figs. 6 and 7(b)-7(e)]. The results reported used bond dimension $D=10$ and the cutoff for the cavities is $n_{\max }=3$. We checked that these sizes are already sufficient for achieving convergence.
[1] O. Astafiev, A. M. Zagoskin, A. A. Abdumalikov, Y. A. Pashkin, T. Yamamoto, K. Inomata, Y. Nakamura, and J. S. Tsai, Science 327, 840 (2010).

[2] I. C. Hoi, C. M. Wilson, G. Johansson, T. Palomaki, B. Peropadre, and P. Delsing, Phys. Rev. Lett. 107, 073601 (2011).

[3] I.-C. Hoi, A. F. Kockum, T. Palomaki, T. M. Stace, B. Fan, L. Tornberg, S. R. Sathyamoorthy, G. Johansson, P. Delsing, and C. M. Wilson, Phys. Rev. Lett. 111, 053601 (2013).

[4] A. F. van Loo, A. Fedorov, K. Lalumière, B. C. Sanders, A. Blais, and A. Wallraff, Science 342, 1494 (2013).

[5] I.-C. Hoi, C. M. Wilson, G. Johansson, J. Lindkvist, B. Peropadre, T. Palomaki, and P. Delsing, New J. Phys. 15, 025011 (2013).

[6] D. Reitz, C. Sayrin, R. Mitsch, P. Schneeweiss, and A. Rauschenbeutel, Phys. Rev. Lett. 110, 243603 (2013).

[7] J. D. Thompson, T. G. Tiecke, N. P. de Leon, J. Feist, A. V. Akimov, M. Gullans, A. S. Zibrov, V. Vuletić, and M. D. Lukin, Science 340, 1202 (2013).

[8] M. Arcari, I. Söllner, A. Javadi, S. Lindskov Hansen, S. Mahmoodian, J. Liu, H. Thyrrestrup, E. H. Lee, J. D. Song, S. Stobbe, and P. Lodahl, Phys. Rev. Lett. 113, 093603 (2014).

[9] R. Yalla, M. Sadgrove, K. P. Nayak, and K. Hakuta, Phys. Rev. Lett. 113, 143601 (2014).

[10] A. Goban, C.-L. Hung, S.-P. Yu, J. D. Hood, J. A. Muniz, J. H. Lee, M. J. Martin, A. C. McClung, K. S. Choi, D. E. Chang, O. Painter, and H. J. Kimble, Nat. Commun. 5, 3808 (2014).

[11] P. Lodahl, S. Mahmoodian, and S. Stobbe, Rev. Mod. Phys. 87, 347 (2015).

[12] A. Javadi, I. Söllner, M. Arcari, S. L. Hansen, L. Midolo, S. Mahmoodian, G. Kiršanske, T. Pregnolato, E. H. Lee, J. D. Song, S. Stobbe, and P. Lodahl, Nat. Comm. 6, 8655 (2015).

[13] J.-T. Shen and S. Fan, Opt. Lett. 30, 2001 (2005).

[14] J.-T. Shen and S. Fan, Phys. Rev. Lett. 95, 213001 (2005).

[15] L. Zhou, Z. R. Gong, Y. X. Liu, C. P. Sun, and F. Nori, Phys. Rev. Lett. 101, 100501 (2008).

[16] E. Rephaeli and S. Fan, Phys. Rev. Lett. 108, 143602 (2012).
[17] K. Koshino, K. Inomata, T. Yamamoto, and Y. Nakamura, Phys. Rev. Lett. 111, 153601 (2013).

[18] K. Inomata, K. Koshino, Z. R. Lin, W. D. Oliver, J. S. Tsai, Y. Nakamura, and T. Yamamoto, Phys. Rev. Lett. 113, 063604 (2014).

[19] E. Sánchez-Burillo, D. Zueco, J. J. García-Ripoll, and L. MartinMoreno, Phys. Rev. Lett. 113, 263604 (2014).

[20] R. W. Boyd, Nonlinear Optics, 2nd ed. (Academic Press, New York, 2003).

[21] Y.-x. Liu, J. Q. You, L. F. Wei, C. P. Sun, and F. Nori, Phys. Rev. Lett. 95, 087001 (2005).

[22] Y.-x. Liu, H.-C. Sun, Z. H. Peng, A. Miranowicz, J. S. Tsai, and F. Nori, Sci. Rep. 4, 7289 (2014).

[23] S. R. Sathyamoorthy, A. Bengtsson, P. Delsing, and G. Johansson, Phys. Rev. A 93, 063823 (2016).

[24] K. Koshino, Phys. Rev. A 79, 013804 (2009).

[25] Y. Chang, A. González-Tudela, C. Sánchez-Muñoz, C. Navarrete-Benlloch, and T. Shi, arXiv:1510.07307.

[26] M. O. Scully and M. S. Zubairy, Quantum Optics (Cambridge University Press, Cambridge, UK, 1997).

[27] P. Král and M. Shapiro, Phys. Rev. Lett. 87, 183002 (2001).

[28] J. Koch, T. M. Yu, J. Gambetta, A. A. Houck, D. I. Schuster, J. Majer, A. Blais, M. H. Devoret, S. M. Girvin, and R. J. Schoelkopf, Phys. Rev. A 76, 042319 (2007).

[29] Y. Chen, C. Neill, P. Roushan, N. Leung, M. Fang, R. Barends, J. Kelly, B. Campbell, Z. Chen, B. Chiaro, A. Dunsworth, E. Jeffrey, A. Megrant, J. Y. Mutus, P. J. J. O’Malley, C. M. Quintana, D. Sank, A. Vainsencher, J. Wenner, T. C. White, M. R. Geller, A. N. Cleland, and J. M. Martinis, Phys. Rev. Lett. 113, 220502 (2014).

[30] É. Dumur, B. Küng, A. K. Feofanov, T. Weissl, N. Roch, C. Naud, W. Guichard, and O. Buisson, Phys. Rev. B 92, 020515 (2015).

[31] B. Peropadre, D. Zueco, D. Porras, and J. J. García-Ripoll, Phys. Rev. Lett. 111, 243602 (2013).

[32] G. Vidal, Phys. Rev. Lett. 91, 147902 (2003). 
[33] G. Vidal, Phys. Rev. Lett. 93, 040502 (2004).

[34] J. J. García-Ripoll, New J. Phys. 8, 305 (2006).

[35] F. Verstraete, V. Murg, and J. I. Cirac, Adv. Phys. 57, 143 (2008).

[36] E. Sánchez-Burillo, J. García-Ripoll, L. Martín-Moreno, and D. Zueco, Faraday Discuss. 178, 335 (2015).

[37] T. Ramos, B. Vermersch, P. Hauke, H. Pichler, and P. Zoller, Phys. Rev. A 93, 062104 (2016).

[38] R. Paškauskas and L. You, Phys. Rev. A 64, 042310 (2001).

[39] A. Muthukrishnan, G. S. Agarwal, and M. O. Scully, Phys. Rev. Lett. 93, 093002 (2004).

[40] D. Zueco, J. J. Mazo, E. Solano, and J. J. García-Ripoll, Phys. Rev. B 86, 024503 (2012).

[41] C. W. Gardiner and M. J. Collett, Phys. Rev. A 31, 3761 (1985).
[42] B. Peropadre, G. Romero, G. Johansson, C. M. Wilson, E. Solano, and J. J. García-Ripoll, Phys. Rev. A 84, 063834 (2011).

[43] Eventually, we will set $r_{M}=-1$; i.e., we neglect losses in the mirror, which is a good experimental assumption.

[44] I. Söllner, S. Mahmoodian, S. L. Hansen, L. Midolo, A. Javadi, G. Kiršansk, T. Pregnolato, H. El-Ella, E. H. Lee, J. D. Song, S. Stobbe, and P. Lodahl, Nat. Nanotechnol. 10, 775 (2015).

[45] S. Fan, Ş. E. Kocabaş, and J.-T. Chen, Phys. Rev. A 82, 063821 (2010).

[46] S. Xu and S. Fan, Phys. Rev. A 91, 043845 (2015).

[47] J. L. Garcia-Palacios, arXiv:cond-mat/0701242.

[48] B. Pirvu, V. Murg, J. Cirac, and F. Verstraete, New J. Phys. 12, 025012 (2010).

[49] M. Suzuki, J. Math. Phys. 32, 400 (1991). 